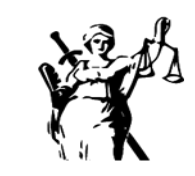

JUSTICIA

ISSN impreso 0124-7441
ISSN digital 2590-4566

\title{
Principios tradicionales del derecho internacional privado con relación a contratos y/o comercio electrónico: caso Unión Europea - Colombia
}

\author{
Traditional principles of private international law in relation to \\ contracts and / or electronic commerce: case of the European \\ Union - Colombia
}

\author{
(i) \\ Carlos Peña Orozco \\ Universidad del Magdalena, Colombia \\ cpena@unimagdalena.edu.co
}

\author{
(iD) \\ Angie Chamorro Buelvas \\ Universidad del Magdalena, Colombia \\ angiechamorromb@unimagdalena.edu.co
}

Recibido: 14 de mayo de 2020 / Aceptado: 26 de julio de 2020

https://doi.org/10.17081/just.26.39.4726

\begin{abstract}
Resumen
En el siguiente artículo, que es producto de una rigurosa revisión documental especializada, se aborda el tema de la solución de conflictos en los contratos electrónicos y cómo se aplica en el derecho comercial internacional, a fin de determinar si efectivamente el uso de los principios tradicionales del Derecho Internacional Privado permite resolver dichos conflictos. En el texto se desarrollan los conceptos de contrato electrónico, contrato inteligente, perfeccionamiento de un contrato electrónico, el impacto que ha tenido en el Derecho, la normatividad que los regula y, finalmente, un ejercicio de derecho comparado entre Colombia y la Unión Europea a fin construir reflexiones de cierre que en lugar de poseer carácter concluyente sugieran futuras líneas de desarrollo e investigación.
\end{abstract}

PALABRAS CLAVE: contrato, consumidor, comercio electrónico, derecho internacional privado.

\begin{abstract}
The following article, which is the product of a rigorous specialized documentary review, addresses the issue of conflict resolution in electronic contracts and how it is applied in international commercial law, in order to determine whether the use of traditional principles of Private International Law allows resolving such conflicts. The text develops the concepts of electronic contract, smart contract, improvement of an electronic contract, the impact it has had on the Law, the regulations that regulate them and, finally, an exercise of comparative law between Colombia and the European Union to In order to construct closing reflections that, instead of being conclusive, suggest future lines of development and research.
\end{abstract}

KEYWORDS: contract, consumer, electronic commerce, private international law.

Como Citar: Peña Orozco, C., \& Chamorro Buelvas, A. (2021). Principios tradicionales del derecho internacional privado con relación a contratos y/o comercio electrónico: caso Unión Europea - Colombia. Justicia, 26(39), 25-36. https://doi.org/10.17081/ just.26.39.4726 


\section{Introducción}

El desarrollo y uso del internet trajo consigo muchos cambios en la cotidianidad de las personas en general, siendo uno de estos el proporcionar un espacio no físico privilegiado desde el cual se pueden adelantar diversas e infinitas tareas y actividades sin tener en cuenta las distancias entre las partes que la realicen. Por ello, si bien la utilización de nuevas tecnologías está presente en el día a día no se puede desconocer que ha traído consigo conflictos propios de su utilización en los cuales se requiere el concurso del Derecho.

Un ejemplo de ello es la realización de contratos electrónicos presentes en las compras de bienes y servicios a nivel local e internacional mediante internet. Este escenario evidencia la problemática que se presenta cuando se trata de contratos entre usuarios de distintas partes del mundo, pues debido a que cada usuario se ciñe a una regulación normativa diferente el contrato sale de la jurisdicción local para ser parte del Derecho Internacional Privado. Queda claro que la situación se antoja compleja "debido en primer lugar al propio medio a través del que se intercambian las voluntades negociables y en segundo extremo, al carácter internacional de muchas de las relaciones que se concluyen en Internet" (Gómez, 2019, p.33).

Esta situación compleja evidencia un panorama desafiante para los estudios jurídicos a la vez que fija como objetivo del presente escrito determinar si los principios tradicionales del Derecho Internacional Privado son suficientes para resolver los conflictos que se presenten en la contratación y/o comercio electrónico (Scotti, 2016) a fin de tener claridad respecto a cuándo se perfecciona el contrato electrónico, poder establecer la competencia al momento de resolver los conflictos generados por este tipo de contratos y explorar las líneas de trabajo en el escenario internacional al respecto toda vez que este tipo de comercio más allá de las fronteras ocupa mucha de la literatura académica en la actualidad, especialmente en países asiáticos (Qi, et al., 2020; Liu \& Li, 2020; Wang, et al., 2020; Sun, et al. 2020) que son considerados los pilares del comercio electrónico.

Para dar cuenta de lo anterior se recurrió a una revisión de textos, escritos científicos y doctrina jurídica, por lo que el escrito inicia por la revisión de la literatura científica que fundamenta el trabajo investigativo documental, se precisarán los conceptos de contrato electrónico, e-commerce y smart contracts, entre otros y se procederá a generar unas reflexiones de cierre a fin de dar cumplimiento al objetivo del escrito.

\section{Comercio electrónico, contrato electrónico y smart contracts}

El concepto de comercio electrónico o e-commerce se refiere a la compra y venta de bienes y servicios a través de medios electrónicos, principalmente la internet como gran plataforma para ello. Esto facilita el comercio entre los usuarios dado que así pueden acceder a una amplia variedad de proveedores, bienes y servicios los cuales cual permiten la comparación de precios, la reducción de costos en las transacciones, entre otros.

Desde sus inicios contemplado como fuente potencial de crecimiento económico a nivel mundial por la manera en que los consumidores han ido cambiando sus hábitos de compra presencial a compras virtuales (Degenhardt et al., 2019), es definido hoy día como una "metodología empresarial moderna que aborda las necesidades de las organizaciones, comerciantes y consumidores para reducir costos al tiempo que mejora la calidad de los bienes y servicios e incrementa la velocidad de la prestación de servicios" (Joseph, 2019, p. 6) que soporta las decisiones tanto de personas naturales como de corporaciones al momento de adquirir bienes y servicios. 
Si bien implican "un modelo de negocios en el cual la actividad comercial se desarrolla en redes electrónicas y en particular, en internet" (Spatari, 2019, p. 4) hay que destacar que "a pesar de su disfraz digital, en este tipo de comercio el contrato, electrónico para el caso, sigue siendo un acuerdo escrito. Si bien es de forma electrónica, su sustancia y ejecución todavía son dependiente de los humanos" (Werbach \& Cornell, 2017, p. 320-321). De allí que se sostiene que si bien esta clase de negocios plantea un reto para el Derecho Internacional Privado, es un reto del cual puede retroalimentarse para bien, en el sentido en que puede adaptarse al escenario complejo y prolijo del comercio electrónico.

Este tipo de comercio que se puede categorizar de acuerdo con la relación entre las partes involucradas y según el tipo de empresa que desarrolla la actividad comercial (Spatari, 2019), ha ido avanzando de manera tal que la empresa Blacksip en su reporte de comercio electrónico anota que los ingresos por este tipo de comercio aumentarían en Colombia en un 35\% para el 2020 con un estimado de 5386 millones de dólares, mientras que en otros países de américa latina como México el aumento sería de $31 \%$ hasta llegar a un estimado de 18.746 millones de dólares y en Perú el aumento estimado era de 40\%. (2020). Por su parte la empresa Nielsen (2018), en un estudio que contempló la revisión de 34 mercados globales, concluía que "para 2022, el comercio electrónico de bienes de consumo masivo aumentará a más de US \$ 400 mil millones y representará alrededor de $10 \%$ a $12 \%$ de las ventas totales de bienes de consumo masivo" (2019, p. 8).

Este tipo de comercio ha sido definido en la Ley 527 de 1999 como toda relación de índole comercial sea o no contractual, estructurada a partir de la utilización de uno o más mensajes de datos o de cualquier otro medio similar. Las relaciones de índole comercial comprenden, sin limitarse a ellas, las siguientes operaciones: toda operación comercial de suministro o intercambio de bienes o servicios; todo acuerdo de distribución; toda operación de representación o mandato comercial; todo tipo de operaciones financieras, bursátiles y de seguros; de construcción de obras; de consultoría; de ingeniería; de concesión de licencias; todo acuerdo de concesión o explotación de un servicio público; de empresa conjunta y otras formas de cooperación industrial o comercial; de transporte de mercancías o de pasajeros por vía aérea, marítima y férrea, o por carretera. (Art 2).

Por lo anterior el legislador reconoce que el comercio electrónico se constituye cuando entre las partes exista una negociación en materia del comercio y que se estructure por medios electrónicos, por lo que se requiere una regulación lo suficientemente amplia que abarque todos los conflictos que puedan surgir de esto, ya sea en el territorio nacional, así como en el extranjero, a fin de colocar límites en este tipo de prácticas contractuales (Khan, 2019).

Esta forma de comercio trajo consigo, entre otras, no solamente los contratos electrónicos sino también una tipología de contratos conocida como smart contracts o contratos inteligentes que se caracterizan por ser una "forma de procesamiento de datos automatizado que promete generar ganancias de eficiencia al mismo tiempo que impulsa nuevos mercados y empresas. Los contratos inteligentes se pueden definir como un código autoejecutable que procesa automáticamente sus entradas cuando se activa" (Finck, 2019, p. 79). Son contratos basados en programación computacional, y fundamentados en el condicional lógico $\mathrm{Si}$... entonces, que poseen la capacidad de ejecutarse una vez que se cumplan unas condiciones concretas (Smart Contract Alliance, 2018). Por lo que no constituye un contrato, sino más bien un recurso computacional que garantiza la ejecución de un contrato una vez corroborado el cumplimiento de todos los pre requisitos previamente establecidos por el programa, que puede ser almacenado en un software que crea varias copias idénticas, es decir, un registro digital. 
Surge la pregunta entonces respecto a si un smart contract podría considerarse, por defecto, un contrato electrónico. Se podría creer que en principio son iguales por los escenarios virtuales en los que se ejecutan, pero en realidad son contratos diferentes pues si bien todo smart contract es un contrato electrónico no significa que todo contrato electrónico sea smart contract. En ese sentido anota Ast que "los contratos inteligentes no son contratos legales. En muchos casos, no pueden ser un buen reemplazo de los contratos tradicionales. Sin embargo, son una nueva herramienta en nuestra limitada caja de herramientas" (2019).

Si bien la idea tras la implementación de smart contracts es la de reducir costos, tiempo de ejecución y acceso a la justicia, buscando con ello promover la eficiencia de los sistemas judiciales, está claro que su implementación en el escenario jurídico implica un replanteamiento epistemológico y una ruptura con la práctica legal tradicional pues este tipo de contratos no se enmarcan en el escenario legal tradicional, ya que en un smart contract se automatiza el proceso de forma tal que la figura del juez no sea requerida. (Ast, 2020).

El comercio electrónico ha generado que en Colombia los establecimientos comerciales se hayan adecuado para complacer a los consumidores, quienes pueden comprar en línea desde productos de la canasta familiar del mercado hasta la adquisición de servicios, logrando con esto importantes oportunidades de empleos para la comunidad y facilitando así el desarrollo de las pequeñas y medianas empresas. Pero también ha posibilitado al consumidor la alternativa de acceder a mercados internacionales al momento de buscar proveedores de ciertos bienes por medio de plataformas virtuales, por lo que esta realidad ha evidenciado ciertas problemáticas y desafíos, algunos clásicos en este tipo de comercio como lo problemático de la interacción mediada por computadoras o la falta de un servicio al cliente personalizado (Tsagkias et al., 2020) y otros relativos a la competencia jurisdiccional, en materia de la normatividad, que se debe aplicar, a la seguridad de los datos, entre otros.

\section{Perfeccionamiento de los contratos electrónicos}

Se entiende que un contrato electrónico se perfecciona cuando existe una oferta y una aceptación de la oferta, además de cumplir con las distintas fases de estas que son: la fase de formación, en la cual existe una oferta y aceptación de esta por medio de la concurrencia de voluntades; la fase de aceptación o adhesión de las condiciones generales del contrato, en el que se determinan los términos del contrato; y finalmente la de ejecución. (Gómez, 2019).

Al respecto Silva-Ruíz amplía este concepto al manifestar que el contrato electrónico es aquel que se perfecciona mediante el intercambio electrónico de datos de ordenador a ordenador. Son los acuerdos de voluntad celebrados a través de medios electrónicos, por los cuales las partes establecen obligaciones exigibles. Dicho contrato se rige por los principios generales de los contratos y las obligaciones de la legislación que le sean aplicables. La noción misma de contrato no se modifica; es el medio el que es sustancialmente diferente al carecer de corporeidad. (2015, p. 231)

Por su parte en el Código Civil Español se estipula sobre el particular que los contratos se perfeccionan a partir del "mero consentimiento, y desde entonces obligan, no sólo al cumplimiento de lo expresamente pactado, sino también a todas las consecuencias que, según su naturaleza, sean conformes a la buena fe, al uso y a la ley" (Art. 1258). Mientras que en el Código Civil Colombiano se mencionan más requisitos para que un contrato se pueda perfeccionar, en síntesis, tiene el mismo concepto que el Código Civil Español, pues al declarar su voluntad se obliga a sí mismo para cumplir con lo pactado. 
Tomando como referente lo anteriormente planteado se da por aceptado un contrato electrónico desde el momento mismo en el que existe una manifestación de la voluntad de querer adherirse a las obligaciones de lo que se ha pactado, teniendo en cuenta las consecuencias de este. Tal consentimiento está ligado a la aceptación de una oferta, es decir, si no existe la oferta no hay consentimiento y sin consentimiento no existe contrato. Por ello el consentimiento es elemento esencial para el perfeccionamiento de cualquier contrato.

Si bien en los contratos tradicionales dicho consentimiento puede manifestarse de manera verbal o escrita, partiendo del hecho que implica la plena certeza de que la persona está declarando su voluntad de aceptar un acuerdo, la validez de tal consentimiento ha generado diversas posiciones y doctrinas en el ámbito jurídico cuando se trata de medios. Esto porque se complejiza la verificación de este al no poder tener la certeza de que la persona natural o jurídica es efectivamente la operante del dispositivo electrónico por medio del cual se está adhiriendo a las obligaciones contractuales.

Lo anterior ha ocasionado algunas formas para expresar la aceptación de un contrato electrónico, de allí que sea necesario mencionar cómo se puede manifestar la voluntad de querer contratar de manera virtual, y entre las formas más comunes para expresar la aceptación de un contrato de esta naturaleza se encuentran las firmas electrónicas definidas por el Decreto 1074 de 2015 como

cualquier método que permita identificar a una persona en relación con un mensaje de datos, tales como códigos, contraseñas, datos biométricos, o claves criptográficas privadas, siempre y cuando el mismo sea confiable y apropiado respecto de los fines para los que se utiliza la firma, atendidas todas las circunstancias del caso, así como cualquier acuerdo pertinente. (Ministerio de Industria, Comercio y Turismo, Art. 2.2.2.47.1)

También se contemplan las firmas digitales, que vendría a ser una especie en el género de las firmas electrónicas, como forma de manifestar la voluntad en un contrato de tipo electrónico. Estas son definidas en la Ley 527 de 1999 como

un valor numérico que se adhiere a un mensaje de datos y que, utilizando un procedimiento matemático conocido, vinculado a la clave del iniciador y al texto del mensaje permite determinar que este valor se ha obtenido exclusivamente con la clave del iniciador y que el mensaje inicial no ha sido modificado después de efectuada la transformación (Art. 2, literal c).

Se reconoce también la posibilidad de aceptar las condiciones generales de un contrato electrónico simplemente haciendo click en una pantalla (Hughes, 2020; Wrigley, 2019, Gómez, 2019), lo que exterioriza la aceptación de la voluntad. Esto ha generado a su vez distintas técnicas que se emplean en los contratos electrónicos para ello, de las cuales se proceden a presentar cuatro.

Una de ellas es el form filling contract que es un tipo de contrato electrónico en el que el usuario debe diligenciar un formulario antes de poder usar los servicios ofertados por un proveedor. En el formulario el usuario entrega información que será utilizada por el proveedor, ejemplo de esto es el diligenciamiento de formularios en las aereolíneas previos al viaje. Con ello el viajero acepta los términos y condiciones de la empresa. (Bhanu \& Saini, 2020).

El click-wrap agreement permite presentar a los contratantes las cláusulas antes o durante el proceso de contratación mediante el sitio web y el consentimiento se produce antes de efectuar el pago (Gómez, 2019). Así el consumidor tiene una posibilidad real de conocer las condiciones del contrato, y de rechazarlas antes de pagar. 
El shrink-wrap agreement es un acuerdo en el producto o servicio objeto del contrato que se recibe con condiciones adicionales no negociables, incluidas en la documentación que le acompaña y que el comprador sólo podrá conocerlas una vez abonado el importe del producto (Gómez, 2019). Por ejemplo, cuando se instala un software, el usuario se obliga de acuerdo con los términos de la licencia que trae el mismo software pero solo se conoce cuando es instalado. Cabe mencionar que para algunos tribunales extranjeros este tipo de aceptación del contrato electrónico no es válido, pues el comprador carece de la posibilidad de conocer dichas condiciones antes de adquirir el producto, ya que estos eliminan completamente la opción de rechazar tales condiciones.

En el browse-wrap agreement la declaración de aceptación de condiciones se produce de forma tácita debido a que en ningún momento se exterioriza la voluntad sólo a través del propio acceso al contenido del sitio que se entiende aceptada con la mera utilización del sitio web, sin necesidad de aceptación expresa. (Gómez, 2019). El simple ingreso al website y la navegación por las distintas opciones mediante links que genera la misma página equivale a la aceptación de condiciones, ejemplo de ello es lo que hacen plataformas de compra y venta como mercadolibre.

\section{Derecho internacional privado y protección al consumidor en contratos electrónicos}

Si bien el Derecho Internacional Privado ofrece las herramientas adecuadas para la regulación de las relaciones contractuales muchas veces no resultan ser suficientes toda vez que dichas normas y/o principios son aplicables en los contratos tradicionales y en especial a la protección del consumidor, surgiendo de esto dudas sobre la manera correcta de llevarlo en práctica. Incluso se plantea que el Derecho Internacional Privado no brinda protección efectiva al consumidor en los contratos transfronterizos por si misma naturaleza neutral (Gillies, 2016).

Como los contratos electrónicos pueden ser realizados entre personas naturales o jurídicas en distintos países esto hace que salgan de la normatividad nacional de las partes para ser asunto del Derecho Internacional Privado, que si bien no tiene una protección especial para los consumidores, si brinda ciertas normas para la solución de conflictos que puedan surgir entre las partes contractuales. Por ejemplo, se pueden solucionar los conflictos que existan en los contratos electrónicos de la misma forma que con los tradicionales al momento de respetar el principio de pacta sunt servanda, es decir, lo pactado obliga y por tal motivo se reconoce a los contratos como ley para las partes, respetando así autonomía de la voluntad.

Al respecto Oviedo (2012) explicaba que por tal motivo se permite la posibilidad a las partes de escoger la ley que será aplicable en los contratos, esto con el fin de evitar los conflictos que pudieran presentarse posteriormente, así mismo elegir el ordenamiento que les resulte más adecuados para resolver sus intereses y satisfacer sus necesidades, siempre y cuando el fin sea escoger una ley neutral en la que no se imponga sobre la ley nacional de cualquiera de las partes. Esto se materializó con la Directiva 2019/771 de la Unión Europea en la que se sentaron las bases para la regulación del comercio electrónico, reconociéndolo como eje del crecimiento económico al interior del mercado europeo. Los planteamientos del legislador al respecto suponen un primer paso en la adaptación del Derecho Privado Europeo a los contratos electrónicos mientras se mantiene la coherencia con la legislación existente (Staudenmayer, 2020).

Aun cuando la regla general que se tiene para los contratos virtuales es aplicar los mismos principios generales y obligaciones que se derivan de estos, los conflictos no se anulan del todo ya que no funcionan apropiadamente con los consumidores una vez aceptan una oferta, dando como origen al contrato. Por ello Gillies manifiesta que "los contratos de consumo desafían la idoneidad de la autonomía de las partes, que es uno de los principios más importantes del Derecho Internacional Privado moderno" (2016) pues existe 
una desigualdad al momento de la negociación que se ve reflejado en las cláusulas del contrato, ya que como menciona la misma autora "una empresa puede insertar unilateralmente una cláusula de elección de ley / foro en un Contrato. Esta cláusula podría ser la que tenga la intención obvia de privar al consumidor de sus derechos legales" (2016, p. 9).

Esto se presenta porque la parte más fuerte del contrato tiende a escoger la ley y ordenamiento jurídico de su país pues es con la que está más familiarizada lo que obliga a los consumidores a litigar en el extranjero. Esto genera un riesgo importante para la parte que ha de "de tener que litigar en otro país con juzgadores que probablemente manejarán criterios nacionales, ignorando los problemas del comercio transfronterizo" (Moreno, 2017) y que se ejemplifica en las relaciones contractuales entre aerolíneas internacionales y viajeros (Pantazi, 2020).

Por tal motivo, la solución de los conflictos contractuales se deja al arbitraje internacional siendo así un punto neutro entre las partes, cuando se demuestra que efectivamente hubo una desigualdad entre las partes al momento de aceptar un contrato. Este tipo de arbitraje se ha constituido como el mecanismo preferido por las empresas transnacionales (Herrera, 2018) tomando de preferencia especialmente a la Cámara de Comercio Internacional; la Corte de Arbitraje Internacional de Londres; la Asociación Americana de Arbitraje; y a la Comisión Interamericana de Arbitraje Comercial. Explica además de que dicho estudio identifica las principales ventajas del Arbitraje Internacional, tales como la flexibilidad procesal que se ajusta a las necesidades de las partes contractuales; la libre elección de árbitros especialistas; entre otros.

\section{Solución de conflictos presentados en los contratos electrónicos: derecho comparado Unión Europea - Colombia}

En Europa a partir de directivas como la 2000/31/CE, la 2011/83/UE, la 2013/524/UE y la 2013/11/UE del Parlamento Europeo y del Consejo se ha establecido que cuando se trata de solución de conflictos entre las partes de un contrato se recurre a formas alternativas para solucionarlo de manera más rápida y sencilla a fin de evitar que se llegue hasta los tribunales. Según lo establecido en la legislación de la Unión Europea los organismos de solución de litigios son evaluados por los Estados miembros, además de que los mismos deben reunir unos requisitos de calidad con el fin de garantizar que actúan de manera efectiva, justa, imparcial, independiente y transparente en los casos de litigio. Normalmente se llega a un resultado antes de 90 días. Así mismo en Europa se cuenta con una plataforma en línea la cual está disponible para resolver litigios entre empresas y consumidores en materia de comercio electrónico nombrada ODR (Online Dispute Resolution)1

Esta plataforma, cuyo funcionamiento es responsabilidad de la Comisión Europea, es muy útil toda vez que es una herramienta eficiente que abre el camino "hacia el acceso asequible a la justicia que el sistema legal presencial no puede ofrecer. El ODR tiene el potencial de exigirle cuentas a las compañías y generar confianza al consumidor en la era digital" (Schmitz, 2020, p. 5) y cuyo uso se busca cumplir con el objetivo de resolución extrajudicial de controversias, ya sean nacionales o internacionales, cuando se refiere a compras realizadas por internet (Nava y Ortega, 2019).

Si bien se cuestiona el hecho de que la plataforma sirve apenas para poner en contacto a las partes, trayendo como consecuencia el que las reclamaciones no sean resueltas lo que pone en duda que sea realmente un mecanismo de solución de conflictos, se puede afirmar que tal plataforma representa un avance significativo en la solución alternativa de conflictos presentados en el comercio electrónico. Si bien

1 Véase https://ec.europa.eu/consumers/odr/main/index.cfm?event=main.home2.show\&lng=ES 
la plataforma es susceptible de ser mejorada no minimiza el hecho de que se ha convertido en una herramienta indispensable para los consumidores de las compras por internet (Tenorio y Scheneider, 2019; Nava y Ortega, 2019).

Así mismo se encuentra la Directiva (UE) 2019/2161 la cual modifica la Directiva 93/13 CEE con la que la Unión Europea buscaba brindar una protección a los consumidores, puntualmente cuando se tratan de cláusulas abusivas en los contratos. Se parte del hecho de que el mundo está cada vez más intercomunicado y que no solamente se pueden abarcar protección a los contratos tradicionales, por ello en su Nuevo Marco para los Consumidores la Comisión Europea extiende dicha protección a los contratos electrónicos para "modernizar las normas actuales y colmar las lagunas en el acervo existente en materia de consumo" (2018, p.4), dando a entender claramente la preocupación de la Unión Europea en actualizar sus normas de protección a consumidores atendiendo a las necesidades del nuevo mundo tecnológico, razón por la cual tanto los consumidores como los comerciantes tendrán que regirse por la nueva directiva y a las sanciones que en ella se estipulan. Así, se consolida una manera de protección mucho más formal pues en el caso de que no se pueda llegar a una solución mediante la plataforma ODR, se pueda resolver mediante el litigio cuya decisión ha de ser de obligatorio cumplimiento.

Por su parte en Colombia respecto a la protección de los consumidores se destacan la Ley 527 de 1999 por medio de la cual se define y reglamenta el acceso y uso de los mensajes de datos, del comercio electrónico y de las firmas digitales, la Ley 1480 de 2011 por medio de la cual se expide el Estatuto del Consumidor y el Decreto 1074 de 2015, por medio del cual se expide el Decreto Único Reglamentario del Sector Comercio, Industria y Turismo.

De manera muy puntual para el caso de la protección al consumidor electrónico se ha regulado por la normatividad del Decreto 1074 del 2015 en el que se definen las ventas a distancia como "las realizadas sin que el consumidor tenga contacto directo con el producto que adquiere, a través de correo, teléfono, catálogo, comercio electrónico o con la utilización de cualquier otra técnica de comunicación a distancia." (Art. 2.2.2.37.6). Así mismo, este Decreto establece los deberes a los productores y proveedores estipulados en la Ley 1480 de 2011, que buscan garantizar la entrega del bien o servicio en la dirección del consumidor previa identificación de este, que el consumidor pueda realizar reclamaciones, devoluciones y seguimiento a su envío, que se tenga toda la información del proveedor del servicio así como la disponibilidad del producto o bien a adquirir y los plazos de entrega (Art. 46).

Por otra parte cuando se trata de las ventas realizadas a través de las redes sociales, tales como Instagram o Facebook, la Superintendencia de Industria y Comercio se ha pronunciado sobre eso por medio del concepto 16-155160-00001-0000 (2016) mencionado que cuando verse sobre asuntos de publicidad engañosas sobre dichas redes sociales, al proveedor y/o productor se le es atribuible las diferentes actuaciones administrativas que establece la SIC, pues si bien la red social no es la culpable de la publicidad engañosa si lo es el proveedor que tiene la responsabilidad de responder por sus actos y por tal motivo procede ante él la respectiva denuncia.

Aunque en el país no existe una plataforma tipo ODR utilizada en la Unión Europea, existen formas alternativas de solución de conflictos tales como la conciliación, arbitraje, mediación, negociación, o amigable composición; a pesar de representar un proceso un poco más complejo que el ofrecido por la plataforma on-line ya mencionado, estos siguen siendo ayudas mucho más rápidas y eficientes que llevar los conflictos hasta la corte. Todo lo anterior, enmarcado además, entre otros en el mandato constitucional contenido en el Artículo 78 de la Constitución Política en virtud del cual, la Ley está llamada a regular el control de calidad de bienes y servicios ofrecidos y prestados a la comunidad, la información que se suministra al 
público en su comercialización, el régimen de responsabilidad de quienes participan en la producción y en la comercialización de bienes y servicios y la garantía de participación que tienen las organizaciones de consumidores y usuarios en el estudio de las disposiciones que les afectan.

\section{Conclusiones}

El consumidor actual, es un consumidor trasfronterizo, cualidad que requiere la procura por eliminar cualquier vicio en el consentimiento en las negociaciones. Si bien el principio de pacta sunt servanda está presente en todos los contratos, incluso en los electrónicos, también se aplican principios tradicionales del Derecho Internacional Privado tales como la autonomía de la voluntad y la libertad contractual. Así, aun cuando en los contratos electrónicos se modifica el medio por el cual se ejecuta el contrato, es decir, que ya no es presencial sino virtual, esto no altera las obligaciones a las cuales las partes están obligadas a cumplir al momento que suscribieron dicho contrato.

El comercio electrónico ha generado desafíos a varios aspectos concernientes al Derecho Internacional Privado tradicional, lo que se ha visto traducido en poca eficiencia al momento de solucionar los retos que este le presenta, como por ejemplo cuando las partes de un contrato electrónico aceptan las condiciones sin comprenderlas totalmente, lo que genera una serie de consecuencias legales para las partes involucradas y poca protección por parte del Derecho Internacional Privado tradicional. Lo anterior porque este no ofrece la regulación adecuada para tratar puntualmente casos de contratación electrónica internacional y/o protección al consumidor cuando versen asuntos de comercio y contratación llevados a cabo en escenarios virtuales, pero si brinda las herramientas necesarias para llegar a un acuerdo entre las partes del litigio por lo que sus principios permiten solucionar los conflictos en cuanto a comercio y contratos de este tipo.

La Unión Europea tiene la ventaja de resolver los conflictos surgidos por compras virtuales mediante una plataforma on-line que facilita la solución de los mismos conflictos, por lo que es un enfoque más armónico y adecuado para el comercio electrónico (Marzorati, 2019). Sin embargo, es poco eficiente cuando el conflicto se prolonga ya que el caso en cuestión es archivado, quedando expuesta su carencia de fuerza coercitiva. En Colombia a pesar de no contar con una plataforma on-line como esta, en materia de protección al consumidor, regulación de contratos, y comercio en internet existen herramientas legales para hacer frente a estos escenarios.

El punto clave estará en que el Derecho Internacional Privado permita hallar un balance entre proveedores y consumidores en el escenario del comercio virtual, teniendo en cuenta, entre otros, la falta de contacto físico y directo entre el consumidor y el producto, lo que demanda de la norma que precisamente regule estos aspectos relacionados con la información y la calidad de la misma de tal forma que esta pueda suplir los defectos que pudieran derivarse de esta falta de contacto. Pero además se requiere un compromiso ético por parte de los comerciantes en procurar el establecimiento de sistemas de información transparentes y de plataformas de pago seguras que generen mayor confianza en el consumidor con el fin no solo de seguir fortaleciendo este sistema sino de lograr pulir esos aspectos del consentimiento que se encuentran en construcción y mejoramiento para las partes. Tal balance está lejos de ser perfecto y de establecerse en un futuro muy cercano pues se requiere una regulación que sea muy simple en su implementación pero que cubra todas las complejidades inherentes este tipo de comercio. Es una línea de trabajo en la cual se realizan avances permanentemente y que de seguro seguirá alimentando iniciativas y trabajos de investigación a futuro. 


\section{Referencias Bibliograficas}

Ast, F. (2019, 17 de julio). Contratos Inteligentes, ¿Por Qué Importan? En https://medium.com/astec/ contratos-inteligentes-por-qué-importan-403381962af1

Ast, F. (2020). Smart Contracts: Nuevos Contratos para la Era Digital. En https://medium.com/astec/smartcontracts-nuevos-contratos-para-la-era-digital-b38b342f076

Bhanu, A. \& Saini, N. (2020). Operational aspects of EContracts: A critical Study. International Journal of Management, 11(10), pp. 1097-1110. En https://www.iaeme.com/MasterAdmin/uploadfolder/ IJM_11_10_099/IJM_11_10_099.pdf

Blacksip. (2020). Reporte de industria del ECommerce: 2020. En https://content.blacksip.com/ebooksreporte-de-industria-del-ecommerce-2020

Código Civil Español, Real Decreto de 24 de julio de 1889, art 1258. En https://www.boe.es/buscar/act. php?id=BOE-A-1889-4763\#art1258

Comisión Europea. (2018). Comunicación de la comisión al parlamento europeo, al consejo y al comité económico y social europeo. Un Nuevo Marco para los Consumidores. En https://eur-lex.europa.eu/ legal-content/ES/TXT/PDF/?uri=CELEX:52018DC0183\&from=ES

Decreto 1074 de 2015. Por medio del cual se expide el Decreto Único Reglamentario del Sector Comercio, Industria y Turismo. 26 de mayo de 2015. En https://www.funcionpublica.gov.co/eva/gestornormativo/ norma.php?i=76608

Jon Degenhardt, J., Kallumadi, S., Porwal, U. \& Trotman, A. (2019). Report on the SIGIR 2019 Workshop on eCommerce (ECOM19). SIGIR Forum, 53:11-19. En https://arxiv.org/abs/1912.12282

Finck, M. (2019). Smart contracts as a form of solely automated processing under the GDPR. International Data Privacy Law, 9(2). pp. 78-94. https://doi.org/10.1093/idpl/ipz004

Gillies, L. (2016). Electronic Commerce and International Private Law: A Study of Electronic Consumer Contracts. Routledge: Londres.

Gomez, E. (2019). El perfeccionamiento del contrato electrónico en derecho internacional privado español. [tesis de doctorado, Universidad de Jaén]. RUJA: Repositorio Institucional de Producción Científica. En http://hdl.handle.net/10953/959

Herrera, K. (2018). Aspectos actuales del arbitraje comercial internacional. Cuadernos de la Maestría en Derecho, (6), 265-328. https://revistas.usergioarboleda.edu.co/index.php/Cuadernos/article/ view/1010

Hughes, G. (2020). Enforceability of Contract Terms Displayed on Social Media. En Jackson, M., \& Shelly, M. (Eds.), Legal Regulations, Implications, and Issues Surrounding Digital Data (pp. 1-22). Pennsylvania: IGI Global. http://doi:10.4018/978-1-7998-3130-3.ch001

Joseph, P.T (2019). E-commerce: an indian perspective. Sexta edición. PHI Learning Pvt: Nueva Delhi.

Khan, L. (2019). The Separation of Platforms and Commerce. 119 Columbia Law Review, 973. En https://ssrn. com/abstract=3180174

Liu, Z. \& Li, Z. (2020). A blockchain-based framework of cross-border e-commerce supply chain. International Journal of Information Management, 52. https://doi.org/10.1016/j.ijinfomgt.2019.102059

Ley 527 de 1999. Por medio de la cual se define y reglamenta el acceso y uso de los mensajes de datos, del comercio electrónico y de las firmas digitales, y se establecen las entidades de certificación y se dictan otras disposiciones. 21 de agosto de 1999. En http://www.secretariasenado.gov.co/senado/ basedoc/ley_0527_1999.html 
Marzorati, O. (2019). Las nuevas tecnologías el impacto de la venta 'on-line' en los contratos frente al ‘blockchain' y los contratos inteligentes. Foro Derecho Mercantil, 65, pp.7-51. En http://legal.legis.com. co/document/Index?obra=rmercantil\&document=rmercantil_99bca5927bdd4a8886bb563746f3fa24

Moreno, J. (2017). Legis. La efectividad del arbitraje comercial internacional como mecanismo de solución de controversias. Foro Derecho Mercantil, 56, pp. 99-127. En http://legal.legis.com.co/document/ Index?obra=rmercantil\&document=rmercantil_ccfc53c2db4b4b58b2dcc243444864f9

Nava, W. \& Ortega, G. (2019). Análisis crítico de la plataforma europea de resolución de conflictos en línea. Revista de Internet, Derecho y Política, 29. pp. 1-15. En https://dialnet.unirioja.es/servlet/ articulo?codigo $=7329216$

Nielsen (2018). Comercio conectado. En https://www.nielsen.com/wp-content/uploads/sites/3/2019/04/ connected-commerce-report-spanish.pdf

Oviedo, J. (2012). Legis. Autonomía de la voluntad en los contratos internacionales. Foro Derecho Mercantil, 35, pp. 11-37. En https://xperta.legis.co/visor/temp_rmercantil_838336f5-2bfe-4e7b-b563-249951424118

Pantazi, T. (2020). I Know that I Know Nothing: Extraordinary Circumstances in Air Passenger Rights Regulation. European Review of Private Law, 28(5), pp. 1093 - 1112. https://kluwerlawonline.com/ journalarticle/European+Review+of+Private+Law/28.5/ERPL2020064

Qi, X., Chan, J., Hu, J. \& Li, Y. (2020). Motivations for selecting cross-border e-commerce as a foreign market entry mode. Industrial Marketing Management, 89, pp. 50-60. https://doi.org/10.1016/j. indmarman.2020.01.009

Schmitz, A. (2020). Promover la protección del consumidor a través de la resolución de disputas en línea. En: Agudelo Mejía, D. A. et al. (Coordinadores académicos). Nuevas dinámicas del Derecho Procesal. Sello editorial Universidad de Medellín: Medellín.

Scotti, L. (2016). El impacto de Internet en el mundo jurídico: Una mirada desde el Derecho Internacional Privado. Foro Jurídico, 15, pp. 178-198. En http://revistas.pucp.edu.pe/index.php/forojuridico/article/ view/19845

Silva-Ruiz, P. (2015, 15 de octubre). La contratación electrónica y el derecho internacional privado. [comunicación]. XXVI Jornadas de la Asociación Española de Profesores de Derecho Internacional y Relaciones Internaciones. Sevilla, España. En http://www.notariado.org/liferay/c/document_library/ get_file?folderld=13807670\&name=DLFE-222973.pdf

Smart Contract Alliance, (2018). Smart Contracts: Is the law ready? En https://digitalchamber.s3.amazonaws. com/Smart-Contracts-Whitepaper-WEB.pdf

Spatari, M. (2019). E-commerce: tendenze recenti e impatto sul lavoro. uni commerce global union. En https://uiltucs.it/wp-content/uploads/2020/01/E-Commerce-Report_Syndex_October_2019_IT.pdf

Staudenmayer, K. (2020). The Directives on Digital Contracts: First Steps Towards the Private Law of the Digital Economy. European Review of Private Law, 28(2), pp. 219 - 250. En https://kluwerlawonline. com/journalarticle/European+Review+of+Private+Law/28.4/ERPL2020012

Sun, L., Lyu, G., Yu, Y. \& Teo, Ch. (2020). Cross-Border E-commerce Data Set: Choosing the Right Fulfillment Option. Manufacturing \& Service Operations Management. https://doi.org/10.1287/msom.2020.0887

Super Intendencia de Industria y Comercio, (26 de julio de 2016). Responsabilidad de las redes sociales en materia de publicidad engañosa [Concepto]. En https://www.sic.gov.co/recursos_user/boletinjuridico-sep2016/conceptos/consumidor/16-0155160-01-del-26-julio-de-2016.pdf 
Tenorio, F. \& Schneider, R. (2019). The online dispute resolution (ODR) in the public administration: the use of technology as an impulse to transparency. Direito, Estado e Sociedade, 54, pp. 171 - 214. En https:// revistades.jur.puc-rio.br/index.php/revistades/article/view/799/550

Tsagkias, M., King, T., Kallumadi, S., Murdck, V. \& Rijke, M. (2020). Challenges and Research Opportunities in eCommerce Search and Recommendations. Sigir Forum, 54(1). En http://sigir.org/forum/issues/ june-2020/

Wang, Y., Jia, F., Schoenherr, T., Gong, Y. \& Chen, L. (2020). Cross-border e-commerce firms as supply chain integrators: The management of three flows. Industrial Marketing Management, 89, pp. 72-88. https:// doi.org/10.1016/j.indmarman.2019.09.004

Werbach, K. \& Cornell, N. (2017). Contracts Ex Machina. Duke Law Journal, 67, pp. 313-382. En https:// scholarship.law.duke.edu/dlj/vol67/iss2/2

Wrigley S. (2019). When People Just Click: Addressing the Difficulties of Controller/Processor Agreements Online. En: Corrales M., Fenwick M., Haapio H. (eds) Legal Tech, Smart Contracts and Blockchain. Perspectives in Law, Business and Innovation. Springer, Singapore. https://doi.org/10.1007/978-98113-6086-2_9 